\title{
Improved criteria for oscillation of noncanonical neutral differential equations of even order
}

\author{
Elmetwally M. Elabbasy ${ }^{1}$, Osama Moaaz' ${ }^{1}$ Higinio Ramos²,3 and Ali Muhib ${ }^{1,4^{*}}$ (D)
}

\section{"Correspondence:}

muhib39@yahoo.com

'Department of Mathematics,

Faculty of Science, Mansoura

University, 35516 Mansoura, Egypt

${ }^{4}$ Department of Mathematics,

Faculty of Education - Al-Nadirah, Ibb University, Ibb, Yemen

Full list of author information is available at the end of the article

\begin{abstract}
In this work, we aim at studying the asymptotic and oscillatory behavior of even-order neutral delay noncanonical differential equations. To the best of our knowledge, most of the related previous works are concerned only with neutral equations in the canonical case. Our new oscillation criteria essentially improve, simplify, and complement related results in the literature, especially those from a paper by Li and Rogovchenko (Abstr. Appl. Anal. 2014:395368, 2014). Some examples are presented that illustrate the importance of the new criteria.
\end{abstract}

MSC: $34 \mathrm{C} 10 ; 34 \mathrm{~K} 11$

Keywords: Neutral differential equations; Even order; Oscillatory behavior; Noncanonical case

\section{Introduction}

Neutral delay differential equations (NDDEs) have many interesting applications in various branches of applied science. It is well known that the modeling of many natural and technological phenomena can be carried out using differential equations, often of a higher order (see [1, 2]). The study of half-linear/Emden-Fowler differential equations with deviating arguments has numerous applications in physics and engineering (e.g., half-linear/Emden-Fowler differential equations arise in the study of $p$-Laplace equations, porous medium problems, chemotaxis models, and so forth); see, e.g., the papers [3, 4] for more details, the papers [5-7] for the oscillation of half-linear differential equations, and the papers $[3,8-10]$ for the oscillation and asymptotic behavior of half-linear/EmdenFowler differential equations with different neutral coefficients.

In this paper, we consider the oscillation and asymptotic behavior of even-order halflinear/Emden-Fowler NDDE of the form

$$
\left(r \cdot\left((u+p \cdot(u \circ \tau))^{(m-1)}\right)^{\alpha}\right)^{\prime}(l)+q(l) u^{\beta}(\sigma(l))=0,
$$

where $l \geq l_{0}, m \geq 4$ is an even integer, $\alpha, \beta$ are ratios of odd positive integers, $r$ is a known differentiable real-valued function, while $p, \tau, q$ and $\sigma$ are known continuous real-valued

(c) The Author(s) 2021. This article is licensed under a Creative Commons Attribution 4.0 International License, which permits use, sharing, adaptation, distribution and reproduction in any medium or format, as long as you give appropriate credit to the original author(s) and the source, provide a link to the Creative Commons licence, and indicate if changes were made. The images or other third party material in this article are included in the article's Creative Commons licence, unless indicated otherwise in a credit line to the material. If material is not included in the article's Creative Commons licence and your intended use is not permitted by statutory regulation or exceeds the permitted use, you will need to obtain permission directly from the copyright holder. To view a copy of this licence, visit http://creativecommons.org/licenses/by/4.0/. 
functions on $\left[l_{0}, \infty\right)$. We also assume that $r(l)>0, r^{\prime}(l) \geq 0, p(l) \in\left[0, p_{0}\right], p_{0}$ is a constant, $q(l) \geq 0, q \neq 0$ on any half-line $[L, \infty)$ for all $L \geq l_{0}, \tau(l) \leq l, \sigma(l) \leq l, \lim _{l \rightarrow \infty} \tau(l)=\infty$, and $\lim _{l \rightarrow \infty} \sigma(l)=\infty$.

To facilitate the analysis and presentation of results, we will define the function $v:=$ $u+p \cdot(u \circ \tau)$. A solution $u(l)$ of (1.1) different from zero means that $u(l)$ is a continuous real-valued function on $\left[l_{0}, \infty\right)$ such that $v \in C^{m-1}\left(\left[l_{0}, \infty\right)\right), r \cdot\left(v^{(m-1)}\right)^{\alpha} \in C^{1}\left(\left[l_{0}, \infty\right)\right)$, and which satisfies the equation in (1.1).

A solution $u(l)$ of (1.1) is called oscillatory if it is neither positive nor negative and presents arbitrarily large zeros on $\left[l_{0}, \infty\right)$; otherwise, it is called nonoscillatory.

Although there are many works that have dealt with the oscillation of solutions of $m$ order neutral differential equations, as far as we know, most of them are concerned only with the canonical operator, that is, when $r(l)$ verifies that

$$
\int_{l_{0}}^{l} r^{-1 / \alpha}(\kappa) \mathrm{d} \kappa \rightarrow \infty \quad \text { as } l \rightarrow \infty
$$

On the other hand, in the noncanonical case, when

$$
\int_{l_{0}}^{\infty} r^{-1 / \alpha}(\kappa) \mathrm{d} \kappa<\infty
$$

the studied equations have the so-called Kneser's solutions. The sign of one of such solutions differs from the sign of its first derivative, that is, $u(l) u^{\prime}(l)<0$. Moreover, in case of even-order differential equations, the assumption (1.2) has been commonly used in the literature to ensure that any possible positive solution $u$ satisfies $u>(1-p) v$, which does not generally hold in the case of (1.3). This results in the difficulty of studying the case when $u(l) u^{\prime}(l)<0$, using the usual techniques (see [11-13]).

From 1969 until recently, the asymptotic behavior of a DDE of the form

$$
\left(r(l)\left(u^{(m-1)}(l)\right)^{\alpha}\right)^{\prime}+q(l) u^{\beta}(\sigma(l))=0,
$$

with the canonical condition in (1.2), has attracted the interest of several authors (see [14-17]). Nonetheless, in 2003 Agarwal et al. [18] obtained a criterion for the existence of a bounded solution of (1.4) under the noncanonical condition (1.3). Later on, Baculikova et al. [19], Li and Rogovchenko [7], and Zhang et al. [20-22] discussed the asymptotic and oscillatory behavior of (1.4) under the condition (1.3). Very recently, Moaaz and Muhib [23] improved and complemented the results in [19-21].

The authors in [24-29] were interested in studying and developing the oscillation theory of even-order neutral equations of the form

$$
(u+p \cdot(u \circ \tau))^{(m)}(l)+q(l) u(\sigma(l))=0 .
$$

To see other oscillation criteria of more general neutral differential equations considering the canonical operator, one can see the references [30,31].

$\mathrm{Li}$ and Rogovchenko [32] obtained some results on the oscillatory and asymptotic behavior of the solutions of (1.1) under the condition (1.3). For the reader's convenience, we present the following result which appeared in [32]. 
Theorem 1.1 Let $m \geq 4$ be even and $0<\alpha=\beta \leq 1$. Assume that $0 \leq p(l) \leq p_{0}<\infty$ for some constant $p_{0}, \tau^{\prime} \geq \tau_{*}>0$, and $\tau \circ \sigma=\sigma \circ \tau$, and there exist three functions $\eta_{1}, \eta_{2}, \eta_{3} \in$ $C\left(\left[l_{0}, \infty\right), \mathbb{R}\right)$ such that

$$
\eta_{1}(l) \leq \sigma(l) \leq \eta_{2}(l), \quad \eta_{1}(l) \leq \tau(l) \leq l<\eta_{2}(l), \quad \eta_{3}(l) \geq \sigma(l), \quad \eta_{3}(l)>l,
$$

and

$$
\lim _{l \rightarrow \infty} \eta_{1}(l)=\infty
$$

Suppose also that

$$
\begin{aligned}
& \frac{\tau_{*}\left(\tau_{*}+p_{0}^{\beta}\right)^{-1}}{((m-1) !)^{\beta}} \liminf _{l \rightarrow \infty} \int_{\tau^{-1}\left(\eta_{1}(l)\right)}^{l} Q(s)\left(\frac{\left(\eta_{1}(s)\right)^{m-1}}{\left(r\left(\eta_{1}(s)\right)\right)^{1 / \beta}}\right)^{\beta} \mathrm{d} s>\frac{1}{\mathrm{e}}, \\
& \frac{\tau_{*}\left(\tau_{*}+p_{0}^{\beta}\right)^{-1}}{((m-2) !)^{\beta}} \liminf _{l \rightarrow \infty} \int_{l}^{\eta_{2}(l)}\left(Q(s)\left(\sigma^{m-2}(s)\right)^{\beta}\left(\delta_{0}\left(\eta_{2}(s)\right)\right)^{\beta}\right) \mathrm{d} s>\frac{1}{\mathrm{e}},
\end{aligned}
$$

and

$$
\frac{\tau_{*}\left(\tau_{*}+p_{0}^{\beta}\right)^{-1}}{((m-3) !)^{\beta}} \liminf _{l \rightarrow \infty} \int_{l}^{\eta_{3}(l)}\left(Q(s)\left(\int_{\eta_{3}(s)}^{\infty}\left(\left(\eta-\eta_{3}(s)\right)^{m-3} \delta_{0}(\eta)\right) \mathrm{d} \eta\right)^{\beta}\right) \mathrm{d} s>\frac{1}{\mathrm{e}},
$$

where $Q(l)=\min \{q(l), q(\tau(l))\}$. Then (1.1) is oscillatory.

In this work, we establish new oscillation criteria for neutral delay differential equations of even order. Unlike most of the previous related works, we are interested in studying the behavior of the solutions of equation (1.1) in the noncanonical case. As far as we know, the unique work related with oscillations in the noncanonical case of (1.1) is [32]. However, in [32], there is no detailed guideline about how to choose the functions $\eta_{i}, i=1,2,3$, fulfilling the forcing conditions, an intriguing issue is how to build up oscillation criteria without requiring the presence of the obscure functions $\eta_{i}$. Here, we will address this topic and introduce some new oscillation criteria. Some examples are provided to illustrate the new results.

The following lemmas are needed in the proofs of our main results.

Lemma 1.1 ([33, Lemma 2.2.3]) Let $f \in C^{m}\left(\left[l_{0}, \infty\right),(0, \infty)\right), f^{(m)}$ be of fixed sign, $f^{(m)} \equiv 0$ on a subray of $\left[l_{0}, \infty\right)$, and $\lim _{l \rightarrow \infty} f(l) \neq 0$. Assume that there is an $l_{1} \in\left[l_{0}, \infty\right)$ such that $f^{(m-1)} f^{(m)} \leq 0$ for $l \in\left[l_{1}, \infty\right)$. Then, there is an $l_{\lambda} \in\left[l_{1}, \infty\right)$ such that

$$
f(l) \geq \frac{\lambda}{(m-1) !} l^{m-1}\left|f^{(m-1)}(l)\right|,
$$

for $\lambda \in(0,1)$ and $l \in\left[l_{\lambda}, \infty\right)$.

Lemma 1.2 ([34]) Assume that $B \geq 0, A>0, \vartheta \geq 0$, and $\mu>0$. Then, we have that

$$
B \vartheta-A \vartheta^{(\mu+1) / \mu} \leq \frac{\mu^{\mu}}{(\mu+1)^{\mu+1}} \frac{B^{\mu+1}}{A^{\mu}} .
$$


Lemma 1.3 ([35, Lemma 1.1]) Assume that $f(l) \in C^{m}\left(\left[l_{0}, \infty\right),(0, \infty)\right)$ and $f^{(m)}(l)$ is eventually of one sign for all large $l$. Then, there exists a nonnegative integer $h \leq m$, with $m+h$ even for $f^{(m)}(l) \geq 0$, or $m+h$ odd for $f^{(m)}(l) \leq 0$, such that

$$
h>0 \quad \text { yields } f^{(k)}(l)>0 \quad \text { for } k=0,1, \ldots, h-1 \text {, }
$$

and

$$
h \leq m-1 \quad \text { yields } \quad(-1)^{h+k} f^{(k)}(l)>0 \quad \text { for } k=h, h+1, \ldots, m-1,
$$

eventually.

\section{Main results}

In order to facilitate the calculation, let us define the following:

$$
\begin{aligned}
& \delta_{0}(l):=\int_{l}^{\infty} r^{-1 / \alpha}(\kappa) \mathrm{d} \kappa, \\
& \eta(l):= \begin{cases}c_{1}^{\beta-\alpha} & \text { if } \alpha \geq \beta, \\
c_{2} \delta_{0}^{\beta-\alpha}(l) & \text { if } \alpha<\beta,\end{cases}
\end{aligned}
$$

and

$$
\mu(l):= \begin{cases}c_{3}^{\beta-\alpha} & \text { if } \alpha \geq \beta, \\ \left(\frac{c_{4}}{(m-3) !} \int_{l}^{\infty}(\varrho-l)^{m-3} \delta_{0}(\varrho) \mathrm{d} \varrho\right)^{\beta-\alpha} & \text { if } \alpha<\beta,\end{cases}
$$

where $c_{1}, c_{2}, c_{3}$, and $c_{4}$ are any positive constants.

Lemma 2.1 Assume that $u(l) \in C\left(\left[l_{0}, \infty\right),(0, \infty)\right)$ is a solution of $(1.1)$. Then $v(l)>0$, $\left(r(l)\left(v^{(m-1)}(l)\right)^{\alpha}\right)^{\prime} \leq 0$, and one of the following cases holds, for $l \in\left[l_{1}, \infty\right), l_{1} \geq l_{0}$ :

(A) $v^{\prime}(l), v^{(m-1)}(l)$ are positive and $v^{(m)}(l)$ is negative;

(B) $v^{\prime}(l), v^{(m-2)}(l)$ are positive and $v^{(m-1)}(l)$ is negative;

(C) $(-1)^{k} v^{(k)}(l)$ are positive for all $k=1,2, \ldots, m-1$.

Proof Assume that $u$ is an eventually positive solution of (1.1). Then, there exists $l_{1} \geq l_{0}$ such that $u(l), u(\tau(l))$, and $u(\sigma(l))$ are positive for all $l \geq l_{1}$. Hence, we see that $v(l)>0$ for $l \geq l_{1}$. It follows from $(1.1)$ that $\left(r(l)\left(v^{(m-1)}(l)\right)^{\alpha}\right)^{\prime} \leq 0$. Now, using Lemma 1.3 with $m$ even, we readily get the cases $(\mathbf{A})-(\mathbf{C})$.

Lemma 2.2 Assume that $u(l) \in C\left(\left[l_{0}, \infty\right),(0, \infty)\right)$ is a solution of $(1.1)$ and that $v(l)$ satisfies (B) in Lemma 2.1. Then $\left(v^{(m-2)}(l)\right)^{\beta-\alpha} \geq \eta(l)$, eventually.

Proof Assume that $u$ is an eventually positive solution of (1.1) and that $v$ satisfies (B) for $l \geq l_{1}$. Let us consider different possibilities.

If we assume firstly that $\alpha=\beta$, then $\left(v^{(m-2)}(l)\right)^{\beta-\alpha}=1$, and the result follows trivially. 
Now, consider that $\alpha>\beta$. Since $v^{(m-2)}(l)$ is a nonincreasing positive function, there is an $m_{1}>0$ such that $v^{(m-2)}(l) \leq m_{1}$, which implies that

$$
\left(v^{(m-2)}(l)\right)^{\beta-\alpha} \geq m_{1}^{\beta-\alpha}
$$

and thus the result holds taking $c_{1}=m_{1}$.

Finally, suppose that $\alpha<\beta$.

Using the decreasingness property of $r\left(v^{(m-1)}\right)^{\alpha}$, we obtain, for $l \geq l_{1}$,

$$
r(l)\left(v^{(m-1)}(l)\right)^{\alpha} \leq r\left(l_{1}\right)\left(v^{(m-1)}\left(l_{1}\right)\right)^{\alpha}=-m_{2}<0,
$$

from which

$$
\left(r^{1 / \alpha} v^{(m-1)}\right)(l) \leq-m_{2}^{1 / \alpha}
$$

Multiplying (2.1) by $r^{-1 / \alpha}(l)$ and integrating it on $[l, L]$, we get

$$
v^{(m-2)}(L) \leq v^{(m-2)}(l)-\int_{l}^{L} \frac{m_{2}^{1 / \alpha}}{r^{1 / \alpha}(\vartheta)} \mathrm{d} \vartheta
$$

Letting $L \rightarrow \infty$, we get

$$
0 \leq v^{(m-2)}(l)-m_{2}^{1 / \alpha} \delta_{0}(l)
$$

that is,

$$
v^{(m-2)}(l) \geq m_{2}^{1 / \alpha} \delta_{0}(l)
$$

Thus, we see that

$$
\left(v^{(m-2)}(l)\right)^{\beta-\alpha} \geq m_{2}^{(\beta-\alpha) / \alpha} \delta_{0}^{\beta-\alpha}(l)=c_{2} \delta_{0}^{\beta-\alpha}(l) .
$$

Therefore,

$$
\left(v^{(m-2)}(l)\right)^{\beta-\alpha} \geq \eta(l) .
$$

The proof is complete.

Lemma 2.3 Assume that $u(l) \in C\left(\left[l_{0}, \infty\right),(0, \infty)\right)$ is a solution of $(1.1)$ and $v$ satisfies condition $(\mathbf{C})$ in Lemma 2.1. Then $v^{\beta-\alpha}(l) \geq \mu(l)$, eventually.

Proof Assuming the hypothesis of the statement in the case $\alpha=\beta$, the result follows readily, as $(v)^{\beta-\alpha}=1$.

Next, we assume that $\alpha>\beta$. Since $v$ is a nonincreasing positive function, there are $M_{3}>0$ and $l_{2} \geq l_{1}$ such that $v \leq M_{3}$, for every $l \geq l_{2}$, and hence

$$
(v)^{\beta-\alpha} \geq M_{3}^{\beta-\alpha}=k_{1} \text {. }
$$


Finally, we suppose that $\alpha<\beta$. Using the decreasingness property of $r\left(v^{(m-1)}\right)^{\alpha}$, we obtain for $l \geq l_{1}$ that

$$
\left(r\left(v^{(m-1)}\right)^{\alpha}\right)(l) \leq\left(r\left(v^{(m-1)}\right)^{\alpha}\right)\left(l_{1}\right)=-M_{4}<0,
$$

which yields

$$
\left(r^{1 / \alpha} v^{(m-1)}\right)(l) \leq-M_{4}^{1 / \alpha}
$$

Multiplying (2.2) by $r^{-1 / \alpha}(l)$ and integrating it on $[l, L]$, we get

$$
v^{(m-2)}(L) \leq v^{(m-2)}(l)-\int_{l}^{L} \frac{M_{4}^{1 / \alpha}}{r^{1 / \alpha}(\vartheta)} \mathrm{d} \vartheta .
$$

Letting $L \rightarrow \infty$ and using $(\mathbf{C})$, we obtain

$$
0 \leq v^{(m-2)}(l)-M_{4}^{1 / \alpha} \delta_{0}(l)
$$

Integrating (2.3) $(m-2)$ times from $l$ to $\infty$, we successively arrive at

$$
\begin{aligned}
& v^{(m-3)}(l) \leq-M_{4}^{1 / \alpha} \int_{l}^{\infty} \delta_{0}(\varrho) \mathrm{d} \varrho \\
& -v^{(m-4)}(l) \leq-M_{4}^{1 / \alpha} \int_{l}^{\infty}\left(\int_{s}^{\infty} \delta_{0}(\varrho) \mathrm{d} \varrho\right) \mathrm{d} s=-\frac{M_{4}^{1 / \alpha}}{1 !} \int_{l}^{\infty}(\varrho-l) \delta_{0}(\varrho) \mathrm{d} \varrho
\end{aligned}
$$

and finally, we get

$$
v(l) \geq \frac{M_{4}^{1 / \alpha}}{(m-3) !} \int_{l}^{\infty}(\varrho-l)^{m-3} \delta_{0}(\varrho) \mathrm{d} \varrho .
$$

Therefore, taking $c_{4}=M_{4}^{1 / \alpha}$, we have that

$$
(v(l))^{\beta-\alpha} \geq \mu(l) .
$$

This completes the proof.

Lemma 2.4 Assume that $u(l) \in C\left(\left[l_{0}, \infty\right),(0, \infty)\right)$ is a solution of $(1.1)$ and $v$ satisfies condition (C) in Lemma 2.1. If

$$
\int_{l_{0}}^{\infty}\left(\int_{l}^{\infty}(v-l)^{m-3}\left(\frac{1}{r(v)} \int_{l_{1}}^{v} q(s) \mathrm{d} s\right)^{1 / \alpha} \mathrm{d} v\right) \mathrm{d} l=\infty
$$

then $\lim _{l \rightarrow \infty} u(l)=0$.

Proof Suppose that $u(l) \in C\left(\left[l_{0}, \infty\right),(0, \infty)\right)$ is a solution of $(1.1)$ and $v$ satisfies condition (C) in Lemma 2.1. Let us denote $\lim _{l \rightarrow \infty} v(l)=D$. We claim that $D=0$. Indeed, for the 
sake of contradiction, suppose that $D>0$, and so for all $\varepsilon>0$ there exists $l_{1} \geq l_{0}$ such that $u(\sigma(l)) \geq D$ for $l \geq l_{1}$. Integrating $(1.1)$ on $\left[l_{1}, l\right]$, we get

$$
r\left(v^{(m-1)}\right)^{\alpha}(l)=r\left(v^{(m-1)}\right)^{\alpha}\left(l_{1}\right)-\int_{l_{1}}^{l} q(s) u^{\beta}(\sigma(s)) \mathrm{d} s \leq-D^{\beta} \int_{l_{1}}^{l} q(s) \mathrm{d} s,
$$

that is,

$$
v^{(m-1)}(l)<-D^{\beta / \alpha}\left(\frac{1}{r(l)} \int_{l_{1}}^{l} q(s) \mathrm{d} s\right)^{1 / \alpha} .
$$

Integrating (2.5) twice on $[l, \infty)$, we successively have that

$$
-v^{(m-2)}(l)<-D^{\beta / \alpha} \int_{l}^{\infty}\left(\frac{1}{r(v)} \int_{l_{1}}^{v} q(s) \mathrm{d} s\right)^{1 / \alpha} \mathrm{d} v
$$

and finally,

$$
\begin{aligned}
v^{(m-3)}(l) & <-D^{\beta / \alpha} \int_{l}^{\infty} \int_{s}^{\infty}\left(\frac{1}{r(v)} \int_{l_{1}}^{v} q(s) \mathrm{d} s\right)^{1 / \alpha} \mathrm{d} v \mathrm{~d} s \\
& =-D^{\beta / \alpha} \int_{l}^{\infty}(v-l)\left(\frac{1}{r(v)} \int_{l_{1}}^{v} q(s) \mathrm{d} s\right)^{1 / \alpha} \mathrm{d} v .
\end{aligned}
$$

Similarly, integrating the above inequality $(m-4)$ times on $[l, \infty)$, we obtain

$$
v^{\prime}(l)<-D^{\beta / \alpha} \int_{l}^{\infty}(v-l)^{m-3}\left(\frac{1}{r(v)} \int_{l_{1}}^{v} q(s) \mathrm{d} s\right)^{1 / \alpha} \mathrm{d} v
$$

Integrating this inequality on $\left[l_{1}, \infty\right)$, we find

$$
v\left(l_{1}\right)>D^{\beta / \alpha} \int_{l_{1}}^{\infty}\left(\int_{l}^{\infty}(v-l)^{m-3}\left(\frac{1}{r(v)} \int_{l_{1}}^{v} q(s) \mathrm{d} s\right)^{1 / \alpha} \mathrm{d} v\right) \mathrm{d} l
$$

which is a contradiction to (2.4). Thus, $D=0$; moreover, the inequality $u \leq v$ implies $\lim _{l \rightarrow \infty} u(l)=0$. The proof is complete.

Theorem 2.1 Let $0 \leq p(l)<1$, and let us assume that (2.4)holds. If the first-order DDE

$$
y^{\prime}(l)+q(l)\left(\frac{\lambda_{0}(1-p(\sigma(l))) \sigma^{m-1}(l)}{(m-1) ! r^{1 / \alpha}(\sigma(l))}\right)^{\beta} y^{\beta / \alpha}(\sigma(l))=0
$$

is oscillatory for some constant $\lambda_{0} \in(0,1)$ and

$$
\limsup _{l \rightarrow \infty} \int_{l_{0}}^{l}\left(\eta(s) q(s)(1-p(\sigma(s)))^{\beta}\left(\frac{\lambda_{1} \sigma^{m-2}(s)}{(m-2) !}\right)^{\beta} \delta_{0}^{\alpha}(s)-\frac{\alpha^{\alpha+1} r^{-1 / \alpha}(s)}{(\alpha+1)^{\alpha+1} \delta_{0}(s)}\right) \mathrm{d} s=\infty
$$

holds for some constant $\lambda_{1} \in(0,1)$, then every solution of (1.1) is either oscillatory or converges to zero as $l \rightarrow \infty$. 
Proof Suppose on the contrary that there is a nonoscillatory solution $u$ of (1.1). Then, we can assume $u(l), u(\tau(l))$, and $u(\sigma(l))$ are positive for $l \geq l_{1} \geq l_{0}$. It follows from Lemma 2.1 that there are three possible cases for the behavior of $v$ and its derivatives.

First, suppose that case (A) holds. From the definition of $v$, we see that

$$
u(l)=v(l)-p(l) u(\tau(l)) \geq(1-p(l)) v(l)
$$

which, together with (1.1), gives

$$
\left(r\left(v^{(m-1)}\right)^{\alpha}\right)^{\prime}(l) \leq-q(l)(1-p(\sigma(l)))^{\beta} v^{\beta}(\sigma(l)) .
$$

From Lemma 1.1, we have

$$
v(l) \geq \frac{\lambda l^{m-1}}{(m-1) !} v^{(m-1)}(l)
$$

for every $\lambda \in(0,1)$. From (2.10) and (2.9), we obtain

$$
\left(r\left(v^{(m-1)}\right)^{\alpha}\right)^{\prime}(l)+q(l)(1-p(\sigma(l)))^{\beta}\left(\frac{\lambda \sigma^{m-1}(l)}{(m-1) !}\right)^{\beta}\left(v^{(m-1)}(\sigma(l))\right)^{\beta} \leq 0 .
$$

Letting $y(l)=r(l)\left(v^{(m-1)}(l)\right)^{\alpha}$, clearly, $y(l)$ is a positive solution of the first-order delay differential inequality

$$
y^{\prime}(l)+q(l)\left(\frac{\lambda(1-p(\sigma(l))) \sigma^{m-1}(l)}{(m-1) ! r^{1 / \alpha}(\sigma(l))}\right)^{\beta} y^{\beta / \alpha}(\sigma(l)) \leq 0
$$

It follows from [36, Theorem 1] that the corresponding differential equation (2.6) also has a positive solution for all $\lambda_{0} \in(0,1)$, which is a contradiction.

Next, consider that case (B) holds. We define the function $\Phi$ by

$$
\Phi:=\frac{r\left(v^{(m-1)}\right)^{\alpha}}{\left(v^{(m-2)}\right)^{\alpha}} .
$$

Then $\Phi(l)<0$ for $l \geq l_{1}$. Noting that $\left(r\left(v^{(m-1)}\right)^{\alpha}\right)^{\prime} \leq 0$, we have

$$
r^{1 / \alpha}(s) v^{(m-1)}(s) \leq r^{1 / \alpha}(l) v^{(m-1)}(l), \quad s \geq l \geq l_{1} .
$$

Multiplying (2.13) by $r^{-1 / \alpha}(s)$ and integrating it on $[l, \infty)$, we obtain

$$
0 \leq v^{(m-2)}(l)+r^{1 / \alpha}(l) v^{(m-1)}(l) \delta_{0}(l)
$$

that is,

$$
-\frac{r^{1 / \alpha}(l) v^{(m-1)}(l) \delta_{0}(l)}{v^{(m-2)}(l)} \leq 1
$$

which in view of (2.12) may be written as

$$
-\Phi(l) \delta_{0}^{\alpha}(l) \leq 1
$$


Differentiating (2.12), we have

$$
\Phi^{\prime}(l)=\frac{\left(r\left(v^{(m-1)}\right)^{\alpha}\right)^{\prime}(l)}{\left(v^{(m-2)}(l)\right)^{\alpha}}-\frac{\alpha r(l)\left(v^{(m-1)}(l)\right)^{\alpha+1}}{\left(v^{(m-2)}(l)\right)^{\alpha+1}},
$$

which, in view of (1.1) and (2.12), becomes

$$
\Phi^{\prime}(l)=-\frac{q(l) u^{\beta}(\sigma(l))}{\left(v^{(m-2)}(l)\right)^{\alpha}}-\frac{\alpha \Phi^{(\alpha+1) / \alpha}(l)}{r^{1 / \alpha}(l)} .
$$

Taking into account the fact that $v^{\prime}(l)>0$ and the definition of $v(l)$, we get that (2.8) holds. Hence, (2.15) becomes

$$
\Phi^{\prime}(l) \leq-\frac{q(l)(1-p(\sigma(l)))^{\beta} v^{\beta}(\sigma(l))}{\left(v^{(m-2)}(l)\right)^{\alpha}}-\frac{\alpha \Phi^{(\alpha+1) / \alpha}(l)}{r^{1 / \alpha}(l)} .
$$

From Lemma 1.1, we find

$$
v(l) \geq \frac{\lambda l^{m-2}}{(m-2) !} v^{(m-2)}(l)
$$

for all sufficiently large $l$ and for every $\lambda \in(0,1)$. Then, (2.16) becomes

$$
\begin{aligned}
\Phi^{\prime}(l) \leq & -q(l)(1-p(\sigma(l)))^{\beta}\left(\frac{\lambda \sigma^{m-2}(l)}{(m-2) !}\right)^{\beta}\left(v^{(m-2)}(\sigma(l))\right)^{\beta-\alpha} \frac{\left(v^{(m-2)}(\sigma(l))\right)^{\alpha}}{\left(v^{(m-2)}(l)\right)^{\alpha}} \\
& -\frac{\alpha \Phi^{(\alpha+1) / \alpha}(l)}{r^{1 / \alpha}(l)} .
\end{aligned}
$$

Since $l \geq \sigma(l)$ and $v^{(m-2)}(l)$ is decreasing, in view of the definition of $\eta(l)$ and Lemma 2.2, we have that

$$
\Phi^{\prime}(l) \leq-\eta(l) q(l)(1-p(\sigma(l)))^{\beta}\left(\frac{\lambda \sigma^{m-2}(l)}{(m-2) !}\right)^{\beta}-\frac{\alpha \Phi^{(\alpha+1) / \alpha}(l)}{r^{1 / \alpha}(l)}
$$

Multiplying (2.17) by $\delta_{0}^{\alpha}(l)$ and integrating it on $\left[l_{1}, l\right]$, we get

$$
\begin{aligned}
0 \geq & \delta_{0}^{\alpha}(l) \Phi(l)-\delta_{0}^{\alpha}\left(l_{1}\right) \Phi\left(l_{1}\right)+\int_{l_{1}}^{l} \frac{\alpha \delta_{0}^{\alpha-1}(s)}{r^{1 / \alpha}(s)} \Phi(s) \mathrm{d} s+\int_{l_{1}}^{l} \frac{\alpha \delta_{0}^{\alpha}(s)}{r^{1 / \alpha}(s)} \Phi^{(\alpha+1) / \alpha}(s) \mathrm{d} s \\
& +\int_{l_{1}}^{l} \eta(s) q(s)(1-p(\sigma(s)))^{\beta}\left(\frac{\lambda \sigma^{m-2}(s)}{(m-2) !}\right)^{\beta} \delta_{0}^{\alpha}(s) \mathrm{d} s .
\end{aligned}
$$

Setting $A=\delta_{0}^{\alpha}(s) / r^{1 / \alpha}(s), B=\delta_{0}^{\alpha-1}(s) / r^{1 / \alpha}(s)$, and $\vartheta=-\Phi(s)$, and using Lemma 1.2, we get

$$
\int_{l_{1}}^{l}\left(\eta(s) q(s)(1-p(\sigma(s)))^{\beta}\left(\frac{\lambda \sigma^{m-2}(s)}{(m-2) !}\right)^{\beta} \delta_{0}^{\alpha}(s)-\frac{\alpha^{\alpha+1} r^{-1 / \alpha}(s)}{(\alpha+1)^{\alpha+1} \delta_{0}(s)}\right) \mathrm{d} s \leq \frac{\Phi\left(l_{1}\right)}{\delta_{0}^{-\alpha}\left(l_{1}\right)}+1
$$

due to (2.14), which contradicts (2.7).

Finally, suppose that $(\mathbf{C})$ holds. From Lemma 2.4 , we see that $\lim _{l \rightarrow \infty} u(l)=0$, which is a contradiction.

The proof of the theorem is complete. 
Remark Combining Theorem 2.1 and the results reported in the papers $[37,38]$ for equation (2.6), one can obtain various oscillation criteria for equation (1.1) in the case where $\alpha=\beta$.

Theorem 2.2 Let us assume that the first-order $D D E(2.6)$ is oscillatory for some $\lambda_{0} \in(0,1)$ and that (2.7) holds for some $\lambda_{1} \in(0,1)$. If

$$
\tau \circ \sigma=\sigma \circ \tau, \quad \tau^{\prime}(l) \geq \tau_{0}>0, \quad \sigma(l) \leq \tau(l),
$$

and

$$
\limsup _{l \rightarrow \infty}\left(\mu(l) \delta_{m-2}^{\alpha}(l) \int_{l_{0}}^{l} Q(\varrho) \mathrm{d} \varrho\right)>\kappa\left(1+\frac{p_{0}^{\beta}}{\tau_{0}}\right)
$$

where

$$
\begin{aligned}
& \delta_{k+1}(l):=\int_{l}^{\infty} \delta_{k}(\varrho) \mathrm{d} \varrho \text { for } k=0,1, \ldots, m-3, \\
& Q(l):=\min \{q(l), q(\tau(l))\},
\end{aligned}
$$

and $\kappa=1$ if $\beta \in(0,1]$; otherwise, $\kappa=2^{\beta-1}$, then every solution of (1.1) is oscillatory.

Proof We argue by contradiction. Assume to the contrary that there is a nonoscillatory solution $u$ of (1.1). Then, we can assume $u(l), u(\tau(l))$, and $u(\sigma(l))$ are positive for $l \geq l_{1} \geq l_{0}$. It follows from Lemma 2.1 that there are three possible cases for the behavior of $v$ and its derivatives.

The proofs of the cases in which (A) or $(\mathbf{B})$ is fulfilled are similar to those of Theorem 2.1. Suppose that $(\mathbf{C})$ holds. Since $\left(r(l)\left(v^{(m-1)}(l)\right)^{\alpha}\right)^{\prime} \leq 0$, we have that

$$
r(s)\left(v^{(m-1)}(s)\right)^{\alpha}-r(l)\left(v^{(m-1)}(l)\right)^{\alpha} \leq 0 \quad \text { for all } s \geq l
$$

or

$$
v^{(m-1)}(s) \leq r^{1 / \alpha}(l) v^{(m-1)}(l) \frac{1}{r^{1 / \alpha}(s)}
$$

Integrating this inequality from $l$ to $\infty$ and using the fact that $v^{(m-2)}$ is a positive decreasing function, we arrive at

$$
-v^{(m-2)}(l) \leq r^{1 / \alpha}(l) v^{(m-1)}(l) \int_{l}^{\infty} \frac{1}{r^{1 / \alpha}(\varrho)} \mathrm{d} \varrho=r^{1 / \alpha}(l) v^{(m-1)}(l) \delta_{0}(l) .
$$

Taking into account the behavior of the derivatives of $v(l)$ and integrating the last inequality $(m-2)$ times from $l$ to $\infty$, we obtain

$$
(-1)^{k+1} v^{(k)}(l) \leq r^{1 / \alpha}(l) v^{(m-1)}(l) \delta_{m-k-2}(l)
$$

for $k=0,1, \ldots, m-3$. On the other hand, from (1.1) we have

$$
u^{\beta}(\sigma(l))=-\frac{1}{q(l)}\left(r(l)\left(v^{(m-1)}(l)\right)^{\alpha}\right)^{\prime}
$$


and taking into account that $\tau^{\prime}(l) \geq \tau_{0}>0$, we get

$$
\begin{aligned}
u^{\beta}(\sigma(\tau(l))) & =-\frac{1}{\tau^{\prime}(l) q(\tau(l))}\left(r(\tau(l))\left(v^{(m-1)}(\tau(l))\right)^{\alpha}\right)^{\prime} \\
& \leq-\frac{1}{\tau_{0} q(\tau(l))}\left(r(\tau(l))\left(v^{(m-1)}(\tau(l))\right)^{\alpha}\right)^{\prime} .
\end{aligned}
$$

From (2.20) and (2.21), after using [39, Lemma 1], we find that

$$
\begin{aligned}
v^{\beta}(\sigma(l)) & =(u(\sigma(l))+p(\sigma(l)) u(\tau(\sigma(l))))^{\beta} \\
& \leq \kappa\left(u^{\beta}(\sigma(l))+p_{0}^{\beta} u^{\beta}(\sigma(\tau(l)))\right) \\
& \leq-\frac{\kappa}{q(l)}\left(r(l)\left(v^{(m-1)}(l)\right)^{\alpha}\right)^{\prime}-\frac{\kappa p_{0}^{\beta}}{\tau_{0} q(\tau(l))}\left(r(\tau(l))\left(v^{(m-1)}(\tau(l))\right)^{\alpha}\right)^{\prime} \\
& \leq-\frac{\kappa}{Q(l)}\left(r(l)\left(v^{(m-1)}(l)\right)^{\alpha}+\frac{p_{0}^{\beta}}{\tau_{0}} r(\tau(l))\left(v^{(m-1)}(\tau(l))\right)^{\alpha}\right)^{\prime},
\end{aligned}
$$

or

$$
\left(r\left(v^{(m-1)}\right)^{\alpha}+\frac{p_{0}^{\beta}}{\tau_{0}} r(\tau)\left(v^{(m-1)}(\tau)\right)^{\alpha}\right)^{\prime}(l) \leq-\frac{1}{\kappa} Q(l) \nu^{\beta}(\sigma(l)) .
$$

Integrating this inequality from $l_{1}$ to $l$, we obtain

$$
\begin{aligned}
& r(l)\left(v^{(m-1)}(l)\right)^{\alpha}+\frac{p_{0}^{\beta}}{\tau_{0}} r(\tau(l))\left(v^{(m-1)}(\tau(l))\right)^{\alpha} \\
& \quad \leq r\left(l_{1}\right)\left(v^{(m-1)}\left(l_{1}\right)\right)^{\alpha}+\frac{p_{0}^{\beta}}{\tau_{0}} r\left(\tau\left(l_{1}\right)\right)\left(v^{(m-1)}\left(\tau\left(l_{1}\right)\right)\right)^{\alpha}-\frac{1}{\kappa} \int_{l_{1}}^{l} Q(\varrho) v^{\beta}(\sigma(\varrho)) \mathrm{d} \varrho \\
& \quad \leq-\frac{1}{\kappa} v^{\beta}(\sigma(l)) \int_{l_{1}}^{l} Q(\varrho) \mathrm{d} \varrho .
\end{aligned}
$$

Since $\left(r(l) v^{(m-1)}(l)\right)^{\prime} \leq 0$ and $\tau(l) \leq l$, we arrive at

$$
\begin{aligned}
\left(1+\frac{p_{0}^{\beta}}{\tau_{0}}\right) r(l)\left(v^{(m-1)}(l)\right)^{\alpha} & \leq-\frac{1}{\kappa} v^{\beta}(\sigma(l)) \int_{l_{1}}^{l} Q(\varrho) \mathrm{d} \varrho \\
& \leq-\frac{1}{\kappa} v^{\alpha}(l) v^{\beta-\alpha}(l) \int_{l_{1}}^{l} Q(\varrho) \mathrm{d} \varrho,
\end{aligned}
$$

which, in view of Lemma 2.3, gives

$$
\left(1+\frac{p_{0}^{\beta}}{\tau_{0}}\right) r(l)\left(v^{(m-1)}(l)\right)^{\alpha} \leq-\frac{1}{\kappa} \mu(l) v^{\alpha}(l) \int_{l_{1}}^{l} Q(\varrho) \mathrm{d} \varrho .
$$

Finally, from the inequality in (2.19) for $k=0$ and (2.22), we have that

$$
\left(1+\frac{p_{0}^{\beta}}{\tau_{0}}\right) \geq \frac{1}{\kappa} \mu(l) \delta_{m-2}^{\alpha}(l) \int_{l_{1}}^{l} Q(\varrho) \mathrm{d} \varrho,
$$

which is a contradiction to (2.18). This completes the proof. 


\section{Some applications}

Example 3.1 Consider the NDDE

$$
\left(l^{4}\left(u(l)+p_{0} u(a l)\right)^{\prime \prime \prime}\right)^{\prime}+q_{0} u(b l)=0
$$

where $a, b \in(0,1)$ and $q_{0}>0$. Then, we note that

$$
\begin{aligned}
& \alpha=\beta=1, \quad m=4, \quad r(l)=l^{4}, \quad p(l)=p_{0}, \\
& \tau(l)=a l, \quad q(l)=q_{0}, \quad \text { and } \quad \sigma(l)=b l .
\end{aligned}
$$

Therefore, it is easy to verify that

$$
\delta_{0}(l)=\frac{1}{3 l^{3}}, \quad \delta_{1}(l)=\frac{1}{6 l^{2}}, \quad \text { and } \quad \delta_{2}(l)=\frac{1}{6 l} .
$$

Next, to apply Theorem 2.1, we must first check that conditions (2.4), (2.6), and (2.7) are fulfilled. A simple calculus shows that the integral in (2.4) is divergent. After replacing and simplifying, (2.6) becomes

$$
y^{\prime}(l)+q_{0} \frac{\lambda_{0}\left(1-p_{0}\right)}{6 b} \frac{1}{l} y(b l)=0 .
$$

Applying a well-known oscillation result [40, Theorem 2.1.1] to the first-order DDE in (3.2), we obtain immediately that it is oscillatory if

$$
\liminf _{l \rightarrow \infty} \int_{b l}^{l} q_{0} \frac{\lambda_{0}\left(1-p_{0}\right)}{6 b} \frac{1}{s} \mathrm{~d} s>\frac{1}{\mathrm{e}}
$$

that is,

$$
q_{0} \ln \frac{1}{b}>\frac{3 ! b}{\left(1-p_{0}\right) \mathrm{e}}
$$

Now, we note that (2.7) reduces to

$$
\limsup _{l \rightarrow \infty} \int_{l_{0}}^{l}\left(q_{0}\left(1-p_{0}\right) \frac{\lambda_{1} b^{2}}{6}-\frac{3}{4}\right) \frac{1}{s} \mathrm{~d} s=\infty
$$

which is satisfied if

$$
q_{0}>\frac{18}{4\left(1-p_{0}\right) b^{2}}
$$

Thus, if conditions (3.3) and (3.4) hold, then every solution of (3.1) is oscillatory or tends to zero. Moreover, if $q_{0}=\kappa b^{-\kappa}(\kappa+1)(\kappa-1)(2-\kappa)\left(p_{0} a^{\kappa}+1\right)$ with $\kappa \in(-1,0)$, then it is easy to verify that $u(l)=l^{\kappa}$ is a nonoscillatory solution of (3.1) and tends to zero as $l \rightarrow \infty$.

On the other hand, to apply Theorem 2.2, we see that the condition (2.18) becomes

$$
\limsup _{l \rightarrow \infty}\left(\frac{1}{6 l} \int_{l_{0}}^{l} q_{0} \mathrm{~d} \varrho\right)>\left(1+\frac{p_{0}}{a}\right)
$$


and so

$$
q_{0}>6\left(1+\frac{p_{0}}{a}\right)
$$

Thus, if conditions (3.3), (3.4), and (3.5) hold, then every solution of (3.1) is oscillatory.

Example 3.2 Consider the NDDE

$$
\left(\mathrm{e}^{3 l}\left(\left(u(l)+\left(1-\frac{1}{l^{2}}\right) u(l-a)\right)^{\prime \prime \prime}\right)^{3}\right)^{\prime}+q_{0} \mathrm{e}^{3 l} u^{3}(l-b)=0,
$$

where $l \geq 1,0<a<b$, and $q_{0}>0$. Then, we note that $\alpha=\beta=3, m=4$,

$$
r(l)=\mathrm{e}^{3 l}, \quad p(l)=1-1 / l^{2}, \quad \tau(l)=l-a, \quad q(l)=q_{0} \mathrm{e}^{3 l}, \quad \text { and } \quad \sigma(l)=l-b .
$$

Therefore, it is easy to verify that

$$
\delta_{i}(l)=\mathrm{e}^{-l} \quad \text { for } i=0,1,2 .
$$

A simple calculus shows that in this case the integral in (2.4) diverges. After replacing and simplifying, (2.6) becomes

$$
y^{\prime}(l)+q_{0} \mathrm{e}^{3 l}\left(\lambda_{0} \frac{(l-b)}{3 ! \mathrm{e}^{l-b}}\right)^{3} y(l-b)=0 .
$$

Applying a well-known oscillation result [40, Theorem 2.1.1], we see that (3.7) is oscillatory. Moreover, (2.7) reduces to

$$
\limsup _{l \rightarrow \infty} \int_{l_{0}}^{l}\left(q_{0} \frac{\lambda_{1}^{3}}{2^{3}}-\left(\frac{3}{4}\right)^{4}\right) \mathrm{d} s=\infty
$$

which is satisfied if $q_{0}>81 / 32$. Thus, every solution of (3.6) is oscillatory or tends to zero if $q_{0}>81 / 32$.

On the other hand, to apply Theorem 2.2, we see that the condition (2.18) becomes

$$
\limsup _{l \rightarrow \infty} \mathrm{e}^{-3 l} \int_{l_{0}}^{l} q_{0} \mathrm{e}^{3(s-a)} \mathrm{d} s>2^{3},
$$

that is, $q_{0}>24 \mathrm{e}^{3 a}$. Thus, every solution of (3.6) is oscillatory if $q_{0}>\max \left\{24 \mathrm{e}^{3 a}, 81 / 32\right\}$.

Acknowledgements

The authors are grateful to the editors and two anonymous referees for a very thorough reading of the manuscript and for pointing out some inaccuracies.

Funding

Not applicable.

Availability of data and materials

Not applicable (no datasets were produced). 
Authors' contributions

All authors contributed equally to this work. All authors read and approved the final manuscript.

\section{Author details}

'Department of Mathematics, Faculty of Science, Mansoura University, 35516 Mansoura, Egypt. ${ }^{2}$ Scientific Computing Group, Universidad de Salamanca, Plaza de la Merced, 37008 Salamanca, Spain. ${ }^{3}$ Escuela Politécnica Superior de Zamora, Avda. Requejo 33, 49029 Zamora, Spain. ${ }^{4}$ Department of Mathematics, Faculty of Education - Al-Nadirah, Ibb University, Ibb, Yemen.

\section{Publisher's Note}

Springer Nature remains neutral with regard to jurisdictional claims in published maps and institutional affiliations.

Received: 17 June 2021 Accepted: 31 August 2021 Published online: 08 September 2021

\section{References}

1. Elsgolts, L.E., Norkin, S.B.: Introduction to the Theory and Application of Differential Equations with Deviating Arguments. Academic Press, New York (1973)

2. Hale, J.K.: Theory of Functional Differential Equations. Springer, New York (1977)

3. Bohner, M., Li, T.: Oscillation of second-order $p$-Laplace dynamic equations with a nonpositive neutral coefficient. Appl. Math. Lett. 37, 72-76 (2014)

4. Li, T., Pintus, N., Viglialoro, G.: Properties of solutions to porous medium problems with different sources and boundary conditions. Z. Angew. Math. Phys. 70(3), 86, 1-18 (2019)

5. Chatzarakis, G.E., Džurina, J., Jadlovská, I.: New oscillation criteria for second-order half-linear advanced differential equations. Appl. Math. Comput. 347, 404-416 (2019)

6. Chatzarakis, G.E., Grace, S.R., Jadlovská, I.: On the sharp oscillation criteria for half-linear second-order differential equations with several delay arguments. Appl. Math. Comput. 397, 1-9 (2021)

7. Li, T., Rogovchenko, Y.V.: On asymptotic behavior of solutions to higher-order sublinear Emden-Fowler delay differential equations. Appl. Math. Lett. 67, 53-59 (2017)

8. Agarwal, R.P., Zhang, C., Li, T.: Some remarks on oscillation of second order neutral differential equations. Appl. Math. Comput. 274, 178-181 (2016)

9. Chatzarakis, G.E., Grace, S.R., Jadlovská, I., Li, T., Tunç, E.: Oscillation criteria for third-order Emden-Fowler differential equations with unbounded neutral coefficients. Complexity 2019, Article ID 5691758 (2019)

10. Li, T., Rogovchenko, Y.V.: Oscillation criteria for second-order superlinear Emden-Fowler neutral differential equations Monatshefte Math. 184(3), 489-500 (2017)

11. Muhib, A., Abdeljawad, T., Moaaz, O., Elabbasy, E.M.: Oscillatory properties of odd-order delay differential equations with distribution deviating arguments. Appl. Sci. 10, 5952 (2020)

12. Moaaz, O., Dassios, I., Muhsin, W., Muhib, A.: Oscillation theory for non-linear neutral delay differential equations of third order, Appl. Sci. 10, 4855 (2020)

13. Moaaz, O., Baleanu, D., Muhib, A.: New aspects for non-existence of Kneser solutions of neutral differential equations with odd-order. Mathematics 8(4), 494 (2020)

14. Dahiya, R.S.: Oscillation criteria of even-order nonlinear delay differential equations. J. Math. Anal. Appl. 54, 653-665 (1976)

15. Grace, S.R.: Oscillation theorems for $n$ th-order differential equations with deviating arguments. J. Math. Anal. Appl. 101, 268-296 (1984)

16. Xu, Z., Xia, Y.: Integral averaging technique and oscillation of certain even order delay differential equations. J. Math. Anal. Appl. 292, 238-246 (2004)

17. Zhang, B.G.: Oscillation of even order delay differential equations. J. Math. Anal. Appl. 127, 140-150 (1987)

18. Agarwal, R.P., Grace, S.R., O'Regan, D.: The oscillation of certain higher-order functional differential equations. Math. Comput. Model. 37, 705-728 (2003)

19. Baculikova, B., Dzurina, J., Graef, J.R.: On the oscillation of higher-order delay differential equations. J. Math. Sci. 187(4)، 387-400 (2012)

20. Zhang, C., Li, T., Sun, B., Thandapani, E.: On the oscillation of higher-order half-linear delay differential equations. Appl. Math. Lett. 24, 1618-1621 (2011)

21. Zhang, C., Agarwal, R.P., Bohner, M., Li, T.: New results for oscillatory behavior of even-order half-linear delay differential equations. Appl. Math. Lett. 26, 179-183 (2013)

22. Zhang, C., Li, T., Saker, S.H.: Oscillation of fourth-order delay differential equations. J. Math. Sci. 201(3), 296-309 (2014)

23. Moaaz, O., Muhib, A.: New oscillation criteria for nonlinear delay differential equations of fourth-order. Appl. Math. Comput. 377, $125192(2020)$

24. Baculikova, B., Dzurina, J.: Oscillation theorems for higher order neutral differential equations. Appl. Math. Comput. 219, 3769-3778 (2012)

25. Li, T., Rogovchenko, Y.V.: Oscillation criteria for even-order neutral differential equations. Appl. Math. Lett. 61, 35-41 (2016)

26. Xing, G., Li, T., Zhang, C.: Oscillation of higher-order quasi-linear neutral differential equations. Adv. Differ. Equ. 2011, 45, 1-10 (2011)

27. Zafer, A.: Oscillation criteria for even order neutral differential equations. Appl. Math. Lett. 11, 21-25 (1998)

28. Zhang, Q., Yan, J., Gao, L.: Oscillation behavior of even-order nonlinear neutral differential equations with variable coefficients. Comput. Math. Appl. 59, 426-430 (2010)

29. Agarwal, R.P., Bohner, M., Li, T., Zhang, C.: A new approach in the study of oscillatory behavior of even-order neutral delay differential equations. Appl. Math. Comput. 225, 787-794 (2013)

30. Moaaz, O., Park, C., Muhib, A., Bazighifan, O.: Oscillation criteria for a class of even-order neutral delay differential equations. J. Appl. Math. Comput. 63(1-2), 607-617 (2020) 
31. Moaaz, O., El-Nabulsi, R.A., Bazighifan, O., Muhib, A.: New comparison theorems for the even-order neutral delay differential equation. Symmetry 12(5), 764 (2020)

32. Li, T., Rogovchenko, Y.V.: Asymptotic behavior of higher-order quasilinear neutral differential equations. Abstr. Appl. Anal. 2014, Article ID 395368, 11 pages (2014)

33. Agarwal, R.P., Grace, S.R., O'Regan, D.: Oscillation Theory for Difference and Functional Differential Equations. Kluwer Academic, Dordrecht (2000). https://doi.org/10.1007/978-94-015-9401-1

34. Moaaz, O., Dassios, I., Bazighifan, O., Muhib, A.: Oscillation theorems for nonlinear differential equations of fourth-order. Mathematics 8, 520 (2020)

35. Kiguradze, I., Chanturia, T.: Asymptotic Properties of Solutions of Nonautonomous Ordinary Differential Equations. Mathematics and Its Applications (Soviet Series), vol. 89. Kluwer Academic, Dordrecht (1993) Translated from the 1985 Russian original

36. Philos, C.G.: On the existence of nonoscillatory solutions tending to zero at $\infty$ for differential equations with positive delays. Arch. Math. 36, 168-178 (1981)

37. Chatzarakis, G.E., Jadlovská, I., Li, T.: Oscillations of differential equations with non-monotone deviating arguments. Adv. Differ. Equ. 2019, 233, 1-20 (2019)

38. Chatzarakis, G.E., Li, T.: Oscillation criteria for delay and advanced differential equations with nonmonotone arguments. Complexity 2018, Article ID 8237634 (2018)

39. Thandapani, E., Li, T.: On the oscillation of third-order quasi-linear neutral functional differential equations. Arch. Math. 47, 181-199 (2011)

40. Ladde, G.S., Lakshmikantham, V., Zhang, B.G.: Oscillation Theory of Differential Equations with Deviating Arguments. Dekker, New York (1987)

\section{Submit your manuscript to a SpringerOpen ${ }^{\circ}$ journal and benefit from:}

- Convenient online submission

- Rigorous peer review

- Open access: articles freely available online

- High visibility within the field

- Retaining the copyright to your article

Submit your next manuscript at $\gg$ springeropen.com 